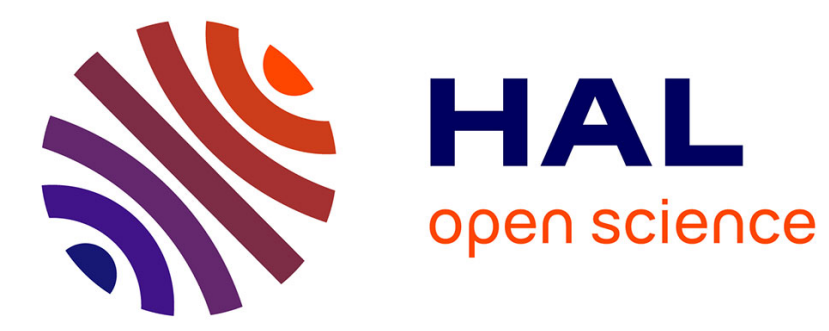

\title{
Les peintures murales du rond-point à Notre-Dame-la-Grande de Poitiers : un programme iconographique et épigraphique très élaboré
}

Robert Favreau

\section{- To cite this version:}

Robert Favreau. Les peintures murales du rond-point à Notre-Dame-la-Grande de Poitiers: un programme iconographique et épigraphique très élaboré. Cahiers de Civilisation Médiévale, 2017, 60 (238), pp.139-154. 10.4000/ccm.1886 . halshs-03206718

\section{HAL Id: halshs-03206718 \\ https://shs.hal.science/halshs-03206718}

Submitted on 23 Apr 2021

HAL is a multi-disciplinary open access archive for the deposit and dissemination of scientific research documents, whether they are published or not. The documents may come from teaching and research institutions in France or abroad, or from public or private research centers.
L'archive ouverte pluridisciplinaire HAL, est destinée au dépôt et à la diffusion de documents scientifiques de niveau recherche, publiés ou non, émanant des établissements d'enseignement et de recherche français ou étrangers, des laboratoires publics ou privés. 


\section{Les peintures murales du rond-point à Notre- Dame-la-Grande de Poitiers : un programme iconographique et épigraphique très élaboré}

Wall-Painting in the Interior of Notre-Dame-la-Grande at Poitiers: A Very Sophisticated Iconographic and Epigraphic Programme

\section{Robert Favreau}

\section{OpenEdition \\ Journals}

Édition électronique

URL : http://journals.openedition.org/ccm/1886

DOI : $10.4000 / \mathrm{ccm} .1886$

ISSN : 2119-1026

Éditeur

Centre d'études supérieures de civilisation médiévale

\section{Édition imprimée}

Date de publication : 1 avril 2017

Pagination : 139-154

ISSN : 0007-9731

\section{Référence électronique}

Robert Favreau, «Les peintures murales du rond-point à Notre-Dame-la-Grande de Poitiers : un programme iconographique et épigraphique très élaboré », Cahiers de civilisation médiévale [En ligne], 238 | 2017, mis en ligne le 01 janvier 2021, consulté le 20 février 2021. URL : http:// journals.openedition.org/ccm/1886 ; DOI : https://doi.org/10.4000/ccm.1886

\section{c) (†) $\odot$}

La revue Cahiers de civilisation médiévale est mise à disposition selon les termes de la Licence Creative Commons Attribution - Pas d'Utilisation Commerciale - Pas de Modification 4.0 International. 


\section{Les peintures murales du rond-point à Notre-Dame-la-Grande de Poitiers : un programme iconographique et épigraphique très élaboré}

\section{RÉSUMÉ}

Des restaurations abusives des peintures murales du rond-point, lors de leur découverte, avec celles de la voûte de l'abside, en 1852, ont conduit à ignorer totalement leur programme iconographique et épigraphique. Deux sibylles, les quatre grands prophètes, les rois David et Salomon, y annoncent la venue du Sauveur célébré dans la voûte, vrai Dieu, le Christ en gloire, et vrai homme, l'Enfant sur les genoux de sa mère. Ce programme s'inspire du Sermon contre les Juifs de Quodvultdeus, qui sera une des sources de l'iconographie de la frise de la façade.

\section{ABSTRACT}

When they were discovered, excessive restorations of the mural paintings of the ambulatory, with those of the vault of the apse in 1852, led to a complete ignorance of their iconographic and epigraphic programmes. Two sibyls, the four Major Prophets and Kings David and Solomon announce the Saviour's arrival celebrated in the vault, true God, Jesus Christ, glory, and true man, the Child in his mother's lap. This programme takes its inspiration from the Homily against the Jews by Quodvultdeus, which is thought to be one of the iconographic sources of the frieze of the façade.

$\mathrm{Au} \mathrm{XIX}^{\mathrm{e}}$ siècle deux architectes, Pierre Gélis-Didot et Henri Laffilée, ont publié un ouvrage sur la peinture décorative en France du $\mathrm{XI}^{\mathrm{e}}$ au XVI ${ }^{\mathrm{e}}$ siècle, où ils présentent côte à côte les dessins très proches de deux prophètes, les bras largement étendus, un phylactère en la main droite, l'un dans la nef de Saint-Savin, l'autre «dans le chœur de Notre-Dame de Poitiers $»^{1}$. Le premier est un des dix prophètes des écoinçons de la nef, identifié par son phylactère: Sicut fuit Jonas in ventre ${ }^{2}$, le second est identifié par son nom, de chaque côté de la tête, IO NAS (fig. 1). Un examen sur place avec des jumelles montrait des rois et d'autres prophètes avec des phylactères partiellement déchiffrables. Les deux auteurs avaient mal lu le nom, qui était celui d'Isaias. Aucun autre auteur n'avait mentionné d'inscription pour les peintures murales du rond-point. Les remarquables photographies prises alors par Jean-Pierre Brouard en août 2016 ont permis de disposer de la totalité du programme iconographique et épigraphique de ces peintures.

1. Pierre Gélis-Didot et Henri Laffilée, La peinture décorative en France du XI au XVI siècle, Paris, s. é., s. d.

2. Corpus des inscriptions de la France médiévale, $1:$ Poitou-Charentes. 2 : Département de la Vienne (excepté la ville de Poitiers), R. Favreau et J. Michaud (éd.), E.-R. Labande (dir.), Poitiers, CNRS/Université de Poitiers, 1975, p. 127-130, fig. 126. 


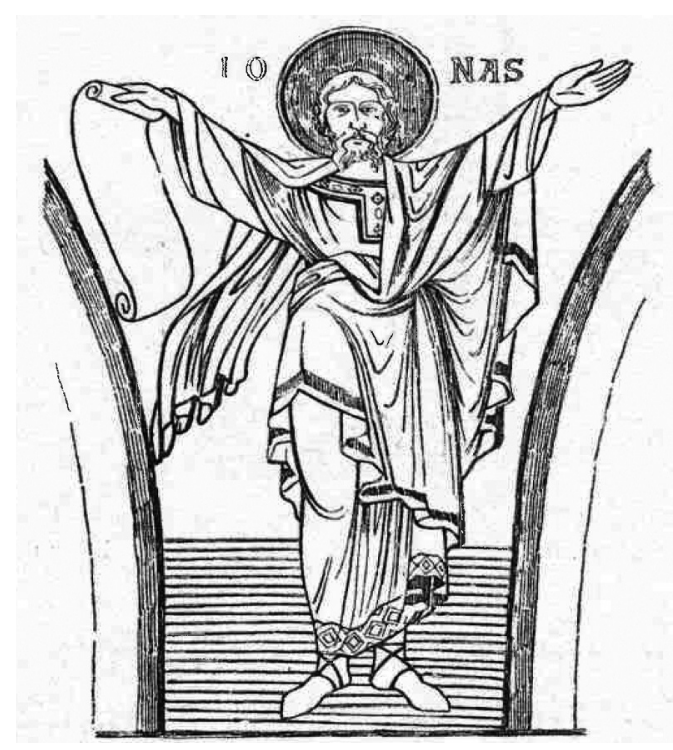

Fig. 1. - Un prophète du rond-point de Notre-Dame-la-Grande, dessin du XIX ${ }^{\mathrm{e}}$ siècle (nom erroné)

L'église Notre-Dame-la-Grande avait été classée monument historique dès 1840. En 1845 Charles JolyLeterme était chargé de la restauration des peintures murales du sanctuaire. Il fit « une copie exacte... des traces visibles et parfaitement distinctes des peintures primitives», et il invita à Poitiers en 1852 le restaurateur Alexandre-Dominique Denuelle ${ }^{3}$. Ce dernier écrit à Prosper Mérimée le 4 décembre 1852: «Nous avons dû préalablement faire enlever les badigeons. Cette opération a mis à découvert une série de peintures à fresque qui pour la plupart sont dans un état presque complet de conservation... Malgré la destruction presque totale des enduits des parties du sanctuaire en contrebas de la corniche nous avons pu constater la présence de figures ailées qui décoraient les tympans, dans l'axe de chacune des colonnes, et encadrées d'une arcature peinte. Dans les pénétrations du bas-côté de droite nous avons retrouvé deux figures de prophètes $»^{4}$. Denuelle a pris pour des "figures ailées» les prophètes qui ont les bras largement étendus, avec un ample drapé de leurs vêtements. Le dessin qu'il a fait des peintures de la voûte de l'abside comprend une figure d'un des écoinçons du rond-point, et on peut constater aujourd'hui que l'arcature peinte qu'il signale n'a pas été conservée au cours de la restauration ${ }^{5}$. La mise au jour des peintures de la voûte après enlèvement des badigeons date précisément de décembre 1852, car Alphonse Letouzey de Longuemar fit dès le début de 1853 une communication à la Société des Antiquaires de l'Ouest «sur les fresques découvertes au mois de décembre 1852 dans l'église Notre-Dame-la-Grande de Poitiers ». La restauration des peintures du rond-point doit dater de cette période, avec des repeints parfois trop appuyés et plusieurs têtes bien trop «revisitées». Les nouvelles restaurations de Charles-Louis Lamaire en 1892, les nettoyages de 1948 et de 1972, semblent n'avoir concerné que la voûte. Le dernier examen, en 2000-2001 n'a porté que sur la voûte. Dans son étude des «peintures de la voûte du chœur de Notre-Dame-la-Grande» en 1951 Paul Deschamps signale en note «qu'aux écoinçons des grandes arcades soutenues par les six colonnes du rond-point on

3. François BERCÉ, «La commission des monuments historiques et la protection des peintures murales sous la monarchie de juillet», dans De fresque en aquarelle. Relevés d'artistes sur la peinture murale romane, catalogue d'exposition (abbaye de Saint-Savin, 2 juillet-10 octobre 1994), S. VAn de Moortele (éd.), Paris, Seuil, 1994, p. 19.

4. Lise Hulnet-Dupuy, «Les peintures murales de la partie orientale. Un chef-d'œuvre méconnu», dans Notre-Dame-la-Grande de Poitiers. L'oeuvre romane, M.-T. Camus et C. Andrault-Schmitt (dir.), Poitiers/Paris, CESCM/Picard, 2002, p. 204-205.

5. Ibid., p. 203, fig. 238. L'auteur dit le dessin de 1845. 
voyait des figures de prophètes bras en croix. Ces figures ont été entièrement repeintes et n'ont donc plus de valeur artistique. Elles avaient les mêmes attitudes que les figures de prophètes très effacées aux écoinçons des arcades de la nef de Saint-Savin $»^{6}$. Yves-Jean Riou en 1995 se borne à indiquer que les prophètes du rond-point ont été totalement repeints au siècle dernier, tout en mentionnant leur parenté avec les prophètes de Saint-Savin ${ }^{7}$. Dans la courte synthèse qu'elle donne sur les peintures murales de Notre-Dame en 1995 Marie-Thérèse Camus écrit: «Dans les écoinçons, des anges qui semblaient porter la voûte ont été remplacés par les prophètes au XIX ${ }^{e}$ siècle, époque à laquelle l'ensemble de l'église fut repeint ${ }^{8}$, et Lise Hulnet-Dupuy porte ce même jugement dans sa thèse de doctorat soutenue à Poitiers en $1996^{9}$. Je pense qu'il y a là une erreur dans l'interprétation de la lettre de Denuelle de 1852 et qu'il n'y a toujours eu que des prophètes au rond-point. On n'a sûrement pas peint au XIX ${ }^{e}$ siècle de nouveaux personnages, et l'on n'était pas en mesure, au milieu du siècle, d'établir un tel programme. L'écriture des inscriptions est, par ailleurs, conforme à ce que l'on sait de la paléographie des inscriptions à la fin $\mathrm{du} \mathrm{XI}^{\mathrm{e}}$ et du début du XII ${ }^{\mathrm{e}}$ siècle : séparation des mots par trois points verticaux, emploi systématique de l'onciale pour le E et le M, mais pas pour d'autres lettres, forme du M, pour autant qu'on puisse appliquer aux peintures murales les observations de Paul Deschamps sur les inscriptions lapidaires ${ }^{10}$, emploi des conjonctions de lettres, à l'exclusion d'abréviation, pour la concision du texte. On n'a jamais jusque-là identifié ces prophètes et les autres figures du rond-point, ni même évoqué la présence de phylactères avec inscriptions. Or avec l'aide des très précieuses photographies dues au perfectionnement des appareils numériques et à la qualité du photographe, on peut suivre aujourd'hui au rond-point un programme très élaboré de deux sibylles à l'ouest, des quatre grands prophètes dans les écoinçons au nord et au sud, des rois David et Salomon dans les écoinçons de l'est, chacune de ces figures avec des inscriptions qui, sauf pour les sibylles, n'ont été que peu ou pas repeintes.

Sur les faces orientales des piliers de la travée qui porte le clocher, c'est-à-dire à l'ouest de l'abside, deux sibylles ont été représentées. Ce sont les figures qui ont été le plus retouchées par la restauration du XIX ${ }^{e}$ siècle. Elles ne sont pas nimbées, car il s'agit de prophétesses païennes, mais leurs prophéties ont eu dès les premiers siècles chrétiens valeur de témoignages incontestés. Émile Mâle dit bien le rôle de la sibylle: «Toute l'antiquité parle par sa bouche... Pendant que les prophètes annonçaient le Messie aux Juifs, la sybille promettait un Sauveur aux païens » ${ }^{11}$. Tertullien dit la sibylle « vraie prophétesse de Dieu». Lactance écrit que «toutes les sibylles prêchent un seul Dieu, principalement la sibylle d'Érythrée qui est considérée comme la plus célèbre et la plus noble des sibylles» ${ }^{12}$. Augustin consacre à la sibylle érythréenne un long passage: «elle a écrit sur le Christ des prophéties évidentes. Nous les avons lues en latin, mauvais latin et vers boiteux... Flaccianus, personnage illustre, qui fut proconsul, homme doué d'une grande facilité de parole et d'un savoir étendu, nous fit voir, un jour que nous parlions ensemble du Christ, un volume grec, en disant que c'étaient les oracles de la sibylle d'Érythrée; il nous y montra un passage où la suite des premières lettres de chaque

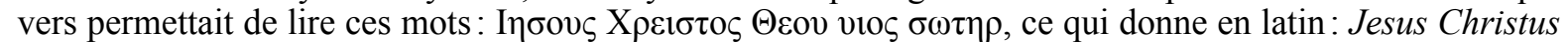
Dei filius Salvator (Jésus Christ Fils de Dieu Sauveur)». Augustin transcrit alors les 27 vers des oracles sibyllins, il rappelle que leurs premières lettres forment un acrostiche, et ajoute que les premières lettres de chaque mot de l'acrostiche forment elles-mêmes le mot $1 \chi \theta u \varsigma$, poisson, «nom qui exprime symboliquement le Christ. Car le Christ, dans l'abîme de cette mortalité semblable aux profondeurs des eaux, a pu rester

6. Congrès archéologique de France, CIX session, Poitiers, en 1951, Paris/Orléans, Société française d'archéologie, 1952, p. 22 , n. 1 .

7. Yves-Jean Riou, Collégiale Notre-Dame-la-Grande, Poitiers, Drac (Itinéraires du patrimoine, 85), 1995, p. 28.

8. Robert Favreau, Marie-Thérèse Camus et François Jeanneau, Notre-Dame-la-Grande, Poitiers, Triolet, 1995, p. 43.

9. Lise Hulnet-Dupuy, Les peintures murales romanes de Notre-Dame-la-Grande à Poitiers, thèse de doctorat en lettres, sous la direction de P. Skubiszewski, Université de Poitiers, 1997, p. 15. Elle ne parle que de la voûte et de la crypte dans l'article qu'elle a publié dans les Cahiers de civilisation médiévale [désormais CCM], XLII, 1993, p. 3-38, avec ce même titre.

10. Paul Deschamps, «Étude sur la paléographie des inscriptions lapidaires de la fin de l'époque mérovingienne aux dernières années du XII siècle», Bulletin monumental, 88, 1929, p. 5-86 (p. 39-44 pour l'étude de la lettre M).

11. Émile MÂLE, L'art religieux du XIII siècle en France. Étude sur l'iconographie du Moyen Âge et sur ses sources d'inspiration [7éd.], Paris, Armand Colin, 1931, p. 339.

12. Tertullien, Apologeticus adversus gentes pro christianis, Patrologie latine [désormais PL], I, c. 385 : vera vates Dei; Lactance, Divinarum institutionum liber primus, (PL, VI, c. 146). 
vivant, c'est-à-dire sans péché» ${ }^{13}$. Mais c'est le Sermon contre les Juifs de son contemporain, l'évêque de Carthage Quodvultdeus, qui aura la plus grande influence dans l'histoire de l'art et son accompagnement épigraphique. Pour convaincre les Juifs que Jésus est le Messie, Quodvultdeus fait intervenir les prophètes, David, le poète Virgile, le roi Nabuchodonosor, la sibylle d'Érythrée, les vers rapportés par Augustin, l'acrostiche grec et sa traduction latine ${ }^{14}$. Ce sermon a dû, évidemment, sa grande fortune au fait qu'il a été attribué à Augustin (jusqu'au $\mathrm{Xx}^{\mathrm{e}}$ siècle). Isidore de Séville indique dans ses Étymologies que sibylle signifie esprit de Dieu, et que les dix sibylles - chiffre généralement retenu - «avaient coutume d'interpréter pour les hommes la volonté divine ${ }^{15}$. Bède le Vénérable rapporte les 27 vers sibyllins de Christo, Paul Diacre dans une homélie sur l'Annonciation s'adresse aux Juifs et copie le Sermon de Quodvultdeus en leur présentant, pour les convaincre, prophètes, Virgile, Nabuchodonosor, et la sibylle dont il cite les 27 vers et l'acrostiche ${ }^{16}$. Fréculphe, évêque de Lisieux, rapporte, au IX $X^{\mathrm{e}}$ siècle, les 27 vers sibyllins et l'acrostiche d'après Augustin ${ }^{17}$. Pierre Abélard cite, d'après la Cité de Dieu d'Augustin, la «fameuse» sibylle érythréenne et l'acrostiche des vers sibyllins ${ }^{18}$. Philippe de Harveng mentionne trois sibylles, dont celle d'Érythrée, la plus importante ${ }^{19}$. Garnier de Rochefort, le cistercien évêque de Langres, cite la sibylle et deux vers sibyllins dans un sermon sur la Nativité de Marie ${ }^{20}$. Jean de Salisbury voit la sibylle annoncer le mystère de l'Incarnation, en se référant à la Cité de Dieu et au célèbre acrostiche des oracles sibyllins ${ }^{21}$. Pierre de Blois reprend, d'après le Sermon, prophètes, sibylles, oracles sibyllins et acrostiche dans son Contra perfidiam Judceorum ${ }^{22}$, et au début du XIII ${ }^{\mathrm{e}}$ siècle, Vincent de Beauvais cite le discours d'Augustin sur la sibylle érythréenne dans son Speculum historiale ${ }^{23}$.

Au pilier nord-est de la travée du clocher de Notre-Dame-la-Grande, à l'entrée de l'abside, est représentée la principale sibylle (fig. 2), avec son nom inscrit au-dessus et de chaque côté de sa tête:

$$
\begin{aligned}
& \text { SYBILLA } \\
& \text { ERY THREA } \\
& \text { Sibylle Érythréenne }
\end{aligned}
$$

Sur le phylactère qu'elle tient dans sa main droite est inscrit le célèbre acrostiche dont parle Augustin, le grec étant maladroitement rendu en caractères latins :
IESO
VS:X
RISTO
S:TEO
$\mathrm{V}: \mathrm{VIO}$
S:SOT
ER:
Jesous Christos Teou Uios Soter
Jésus Christ Fils de Dieu Sauveur

13. Augustin, De civitate Dei, Turnhout, Brepols (Corpus christianorum. Series latina. XI-XXII. Aurelii Augustini opera pars XIV2), 1955, p. 613-614. Le passage est au livre XVIII, c. XXIII. Dom H. LeCLercQ (éd.) parle longuement de ces jeux de lettres dans le Dictionnaire d'archéologie chrétienne et de liturgie, Paris, Letouzey et Ané, I, c. 356-358 ; VII, c. 2001-2002 ; XII, c. 2219 -2244.

14. Opera Quodvultdeo Carthaginiensi episcopo tributa, R. Braun (éd.), Turnhout, Brepols, (Corpus christianorum. Series latina, LX), 1976, p. 248-249.

15. PL, LXXXII, c. 309-310.

16. Ibid., XCV, c. 1473-1474.

17. Ibid., CVI, c. 980-981.

18. Ibid., CLVIII, c. 246-7 (lettre à Héloïse).

19. Ibid., CCIII, c. 1020, De silentio clericorum.

20. Ibid., CCV, c. 770.

21. Ibid., CXCIX, c. 429-430, Polycraticus.

22. Ibid., CCVII, c. 863-871.

23. Vincent DE BeAuvais, Speculum historiale, Douai, B. Bellère, 1624, liber II, caput 50, p. 79-80. 


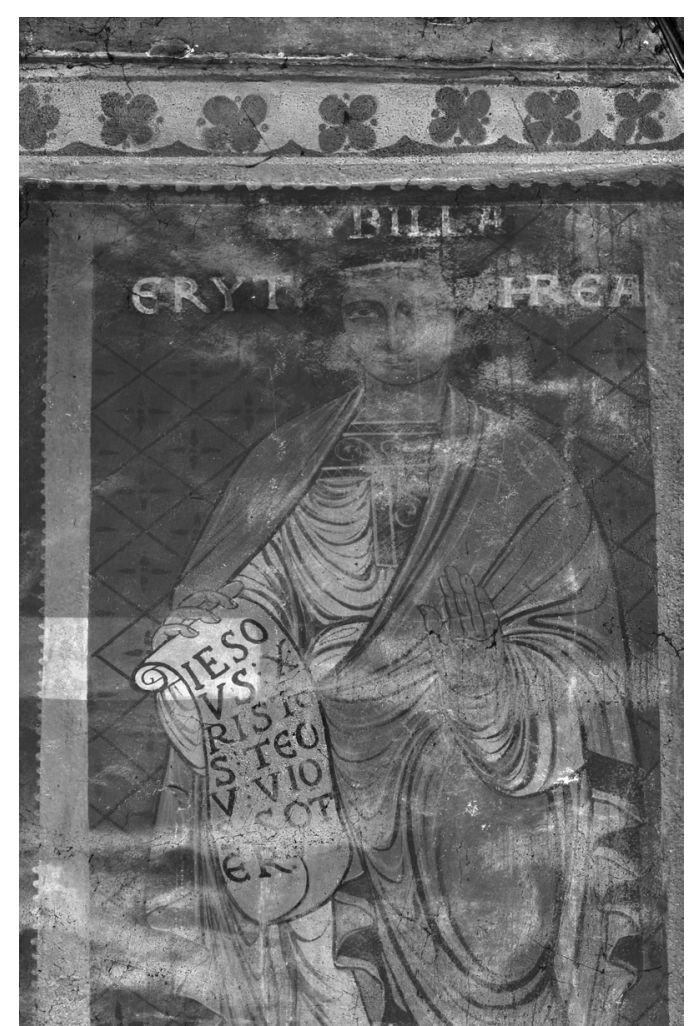

Fig. 2. - La sibylle Erythréenne (cl. CIFM-CESCM/J.-P. Brouard)

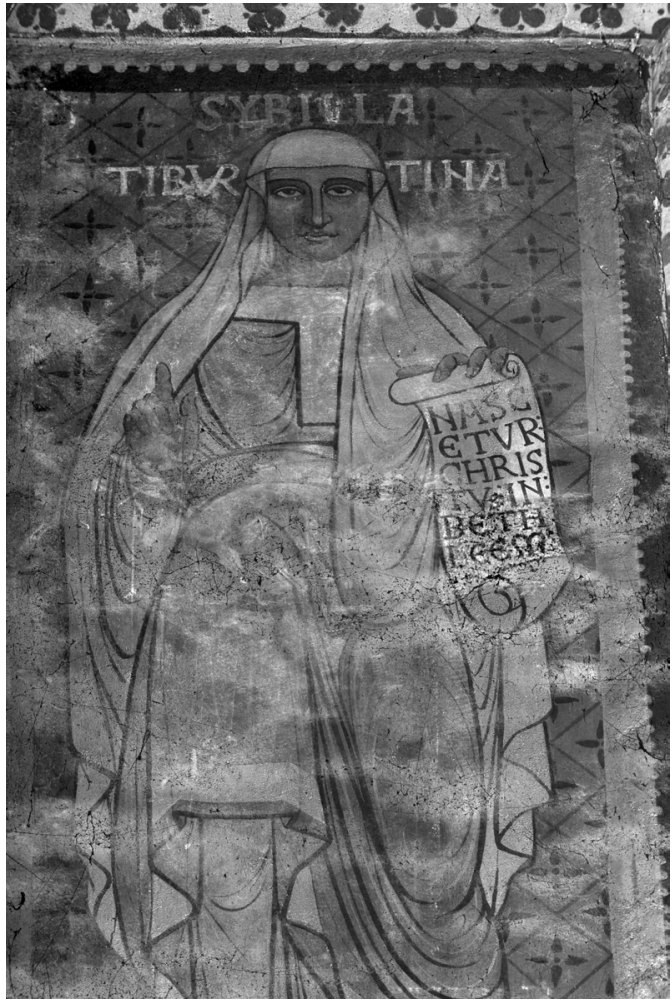

Fig. 3. - La sibylle Tiburtine (cl. CIFM-CESCM/J.-P. Brouard)

Au pilier sud-est de la travée du clocher, donc toujours à l'entrée de l'abside, a été représentée la dernière des dix sibylles, la sibylle Tiburtine (fig. 3), qui était de Tivoli-l'antique Tibur-près de Rome. On lit au-dessus et de chaque côté de sa tête:

SYBILLA

TIBVR TINA

Sibylla Tiburtina

Sur le phylactère qu'elle tient dans la main gauche on lit:

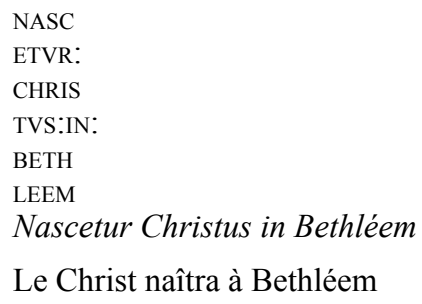

Le Christ naîtra à Bethléem

Bernard Mc Ginn a fait le point sur les origines de cette prédiction de la Nativité du Seigneur par la sibylle Tiburtine, montrant, au Capitole, l'apparition de la Vierge avec l'Enfant à l'empereur Auguste qui lui demandait qui règnerait après lui sur le monde. Auguste aurait alors fait élever sur le capitole, là où il avait entendu la prophétie, un grand autel sur lequel il fit inscrire que c'était «l'autel du Fils de Dieu». Cet autel serait toujours en place, pensait-on, en l'église Santa Maria d'Ara Cœli. Cette légende apparaît dans la 
Chronique de l'Antiochien Jean de Malalas au $\mathrm{VI}^{\mathrm{e}}$ siècle. On la trouve en Occident dans une chronique du $\mathrm{VIII}^{\mathrm{e}}$ siècle ${ }^{24}$, puis au XII ${ }^{\mathrm{e}}$ siècle dans les Mirabilia urbis Romae, et Innocent III y fait référence dans un sermon sur la Nativité du Seigneur ${ }^{25}$. Jacques de Voragine apportera le concours de sa célèbre Légende dorée à cette tradition de la sibylle annonçant à Auguste que l'Enfant porté par une Vierge bien belle serait plus grand que lui ${ }^{26}$. Bernard Mc Ginn indique que le Moyen Âge a utilisé la Sibylle de trois façons : la prophétie de la venue du Christ à l'empereur Auguste, scène souvent évoquée dans l'art et la littérature, les oracles sibyllins et en particulier l'acrostiche, et une troisième façon, «la plus intéressante, [qui] concerne la production de nouveaux textes en prose, apocryphes, attribués à la Sibylle... Les plus importants sont connus sous les noms Sibylla Tiburtina et Sibylla Erythrcea» ${ }^{27}$.

La sibylle Tiburtine représentée à Notre-Dame, moins connue que la sibylle Érythréenne, fait assurément partie de la culture des gens d'Église au XII siècle. Elle sera, elle aussi, à l'honneur lors de la Renaissance italienne et on la trouvera à Rome, avec la même annonce qu'à Notre-Dame-la-Grande, NASCETUR CHRISTUS IN BETHLEem, dans les Chambres Borgia du Vatican, à S. Pietro in Montorio, à Santa Maria sopra Minerve ${ }^{28}$. Le Sermon contre les Juifs de Quodvultdeus, alors attribué à Augustin, a eu une grande fortune de la fin du $\mathrm{XI}^{\mathrm{e}}$ au début du XIII ${ }^{\mathrm{e}}$ siècle, époque où les controverses judéo-chrétiennes sont nombreuses ${ }^{29}$. Il entre dans la liturgie, il sera lu comme sixième leçon de l'office des matines de la Nativité, et l'on voit dans la seconde moitié du Xi ${ }^{e}$ siècle le préchantre de Saint-Martial de Limoges appeler les prophètes -rôles joués par des clercs - à comparaître l'un après l'autre, et chacun s'avancer et chanter son témoignage, celui-là même du Sermon de Quodvultdeus. Ce "drame liturgique» sera joué au XII et XIII siècle dans les cathédrales de Salerne, de Laon et de Rouen. Il sera ensuite joué à l'extérieur, et sera ainsi à l'origine du théâtre ${ }^{30}$. Le Mystère d'Adam, qui intègre la procession des prophètes et les témoignages de David et de Nabuchodonosor sera une source essentielle de la frise de Notre-Dame ${ }^{31}$. Au dernier quart du XIX ${ }^{\mathrm{e}}$ siècle, Marius Sepet a montré que le théâtre du Moyen Âge avait son origine dans les drames liturgiques, et Julien Durand a fait le lien entre le Sermon contre les Juifs, repris dans le Drame des prophètes, et de nombreux programmes iconographiques du XII ${ }^{e}$ siècle $^{32}$. Arthur K. Porter, Émile Mâle, Karl Young populariseront ces études. De fait le Sermon, et en particulier la Procession des prophètes, est illustré, outre la frise de Notre-Dame, aux façades des cathédrales de Crémone, Ferrare, Vérone ${ }^{33}$, d'Orense et de Tarragone, et de bien d'autres œuvres en Italie, en Allemagne, à Jérusalem. En 1233, le pape Grégoire IX interdira aux simples fidèles de participer

24. Chronicon palatinum, $P L$, XCIV, c. 1165, et Chronica minora sae. IV, V, VI, VII, vol. 3, Th. Mommsen (éd.), Berlin, Weidmann (MGH, AA 13), 1898 p. 428-429. Le Repertorium fontium historiae Medii Aevi, III, Isime, Rome, 1970, p. $402-403$ date la chronique du VIII ${ }^{\mathrm{e}}$ siècle. Cette chronique est citée par le chanoine MARSAUX, «La prédiction de la sibylle et la vision d'Auguste», Bulletin Monumental, 70, 1906, p. 236 (235-250).

25. Bernard Mc Ginn, «Teste David cum Sibylla. The Significance of the Sibylinne Tradition in the Middle Ages», Women of the Medieval World. Essays in honor of John H. Mundy, J. Kirshner et S. F. Wemple (éd.), Oxford, B. Blackwell, 1985, p. 21 (p. 7-35). Le Sermon d'Innocent III est dans $P L$, CCXVII, c. 457.

26. Jacopo de VArazzo, Legenda aurea [2 éd.], G.-P. MAGgioni (éd.), Florence, Sismel (Millenio medievale, 6), 1998, p. 69-70.

27. Bernard Mc Ginn, «Sibyllins (oracles)», Dictionnaire encyclopédique du Moyen Âge, A. VAuchez et C. Vincent (dir.), Paris, Le Cerf, II, 1997, p. 1433-1434. ID., «Teste David cum Sibylla» (art. cit. n. 25), p. 24, écrit : «The most popular of these new medieval oracles was the Sybylla Tiburtina, a medieval best-seller, since over 130 Latin manuscripts are known, about 30 before the thirteenth century ». Même si ces nouveaux oracles ne concernent pas le programme de Poitiers, ils attestent que les sibylles étaient bien connues aux $\mathrm{XI}^{\mathrm{e}}$-XII ${ }^{\mathrm{e}}$ siècles.

28. Xavier Barbier de Montault, Iconographie des sibylles, Arras, A. Planque, 1874 (tiré à part de la Revue de l'art chrétien), p. $23-28$.

29. Robert FAVREAU, «Controverses judéo-chrétiennes et iconographie. L'apport des inscriptions », Comptes rendus des séances de l'Académie des Inscriptions et Belles-Lettres, 145 année, $\mathrm{n}^{\circ}$ 3, p. 1267-1303.

30. Karl Young, The Drama of the Medieval Church, t. II, Oxford, Clarendon Press, 1933.

31. Le Mystère d'Adam (Ordo representacione Ade), P. Aebischer (éd), Genève/Paris, Droz/Minard (Textes littéraires français), 1963, date le Mystère de 1220-1250; The Play of Adam (Ordo representacionis Ade), C. J. Odenkirchen (éd.), Brookline, Classical Folia Editions (Medieval classics, 5), 1976, propose 1125-1175.

32. Marius SÉPET, Les prophètes du Christ. Étude sur les origines du théâtre au Moyen Âge, Paris, Didier, 1878 ; Julien DuRAND, «Monuments figurés du Moyen Âge d'après les textes liturgiques», Bulletin monumental, 54, 1888, p. 521-550.

33. Robert Favreau, Épigraphie médiévale, Turnhout, Brepols (L’atelier du médiéviste, 5), 1997 p. 225-230. 


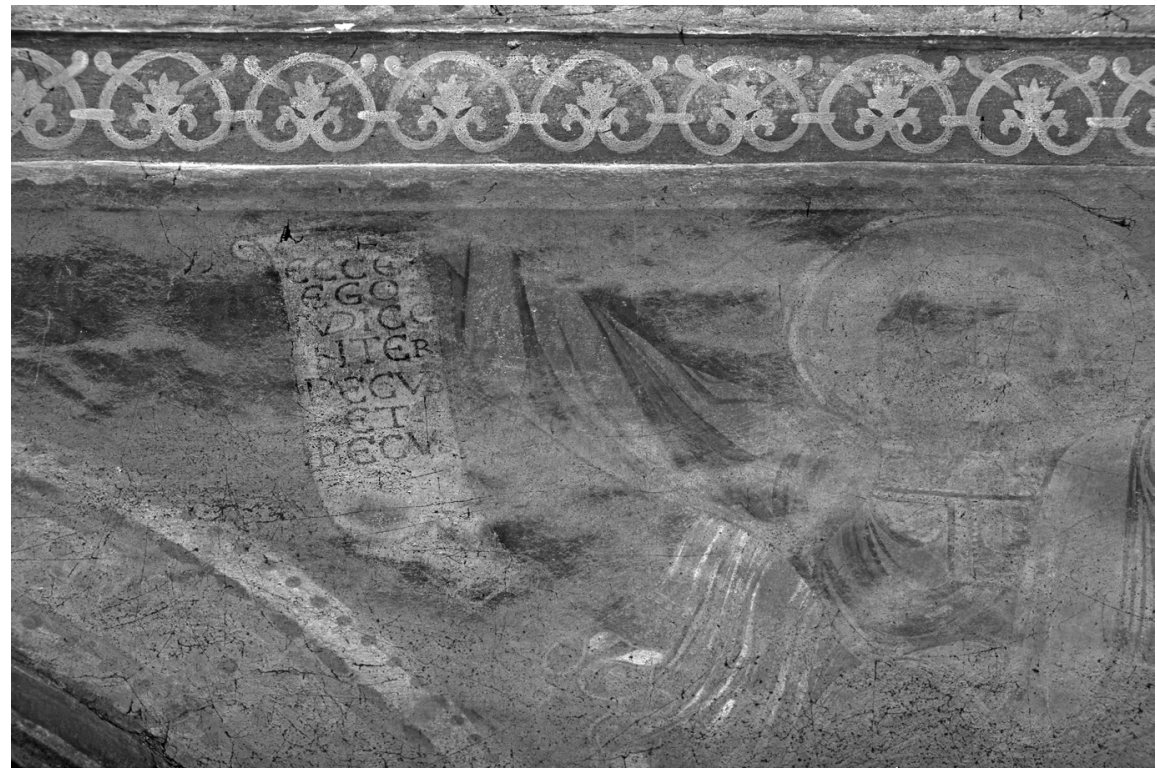

Fig. 4. - Le prophète Ezéchiel

(cl. CIFM-CESCM/J.-P. Brouard)

à des discussions religieuses avec les Juifs, et le débat public ordonné à Paris par le roi Louis IX en 1240, la «dispute» de Barcelone en 1263 n'auront plus que des apparences de discussions. Les Juifs vont être expulsés de France, d'Angleterre, Le Sermon contre les Juifs de Quodvultdeus perd alors de son actualité ${ }^{34}$. Trop retouchées, les figures des sibylles, comme aussi celles des rois David et Salomon, au rond-point de Notre-Dame-la-Grande, n'ont plus de valeur artistique. Mais les quatre grands prophètes peu ou pas retouchés, ont encore belle allure avec leurs bras largement ouverts et l'ample drapé de leur vêtement. Aux écoinçons nord du rond-point sont représentés, de gauche à droite, Ézéchiel et Isaïe.

Le nom d'Ézéchiel (fig. 4) est écrit de chaque côté de sa tête:

EZEC HIEL

Ézéchiel

Sur le phylactère que le prophète tient en sa main droite on lit :

ECCE:

EGO:

IVDICO:

INTER:

PECVS:

ET:

PECVS:

Ecce ego judico inter pecus et pecus

Voici que je vais juger entre brebis et brebis

Cette citation d'Ézéchiel 34:17 est reprise dans Matthieu 25:32, à propos du Jugement dernier. La versio antiqua dit: inter ovem et ovem, et c'est ainsi qu'Augustin cite le verset dans le commentaire qu'il en donne: «Ego judico, les bons sont en sécurité, nul adversaire ne corrompt leur juge, nul avocat ne les abuse, nul

34. Bernard Blumenkranz, «La polémique anti-juive dans l'art chrétien du Moyen Âge », Bulletino dell' Istituto storico italiano per il Medio Evo, 77, 1965, p. 21-44. 
témoin ne se joue d'eux. Mais autant les bons sont en sécurité, autant les mauvais craignent», notamment «ceux qui apprennent dans le calme, mais enseignent de façon trouble» ${ }^{35}$. Le commentaire de saint Jérôme ${ }^{36}$ glose la suite du chapitre 34 : le Seigneur «a parlé contre les pasteurs, maintenant il s'adresse aux brebis et chèvres... et encore béliers et boucs qui sont les chefs parmi le troupeau », à ceux qui ont bien appris les Écritures mais en détournent le sens. «Le Seigneur jugera entre brebis et brebis, non pas en fonction de ces dignités dont s'enorgueillissent les chefs, mais entre homme et homme selon que l'un et l'autre est le serviteur du Seigneur». Jérôme rappelle le verset 23: «Je susciterai pour le mettre à leur tête un pasteur qui les fera paître, mon serviteur David». Raban Maur au IX ${ }^{\mathrm{e}}$ siècle reprendra littéralement saint Jérôme ${ }^{37}$. Ézéchiel n'est pas nommé dans le Sermon contre les Juifs. On l'a retenu à l'évidence pour une raison de symétrie avec les trois autres grands prophètes. Peut-être cette citation a-t-elle été choisie parce qu'elle renvoie au roi David présenté dans l'axe du rond-point et cité par Jérémie, du côté sud, lui aussi dans le premier écoinçon.

Au deuxième écoinçon, Isaïe (fig. 5) est identifié par son nom de part et d'autre de sa tête:

ISA IAS

Isaïe

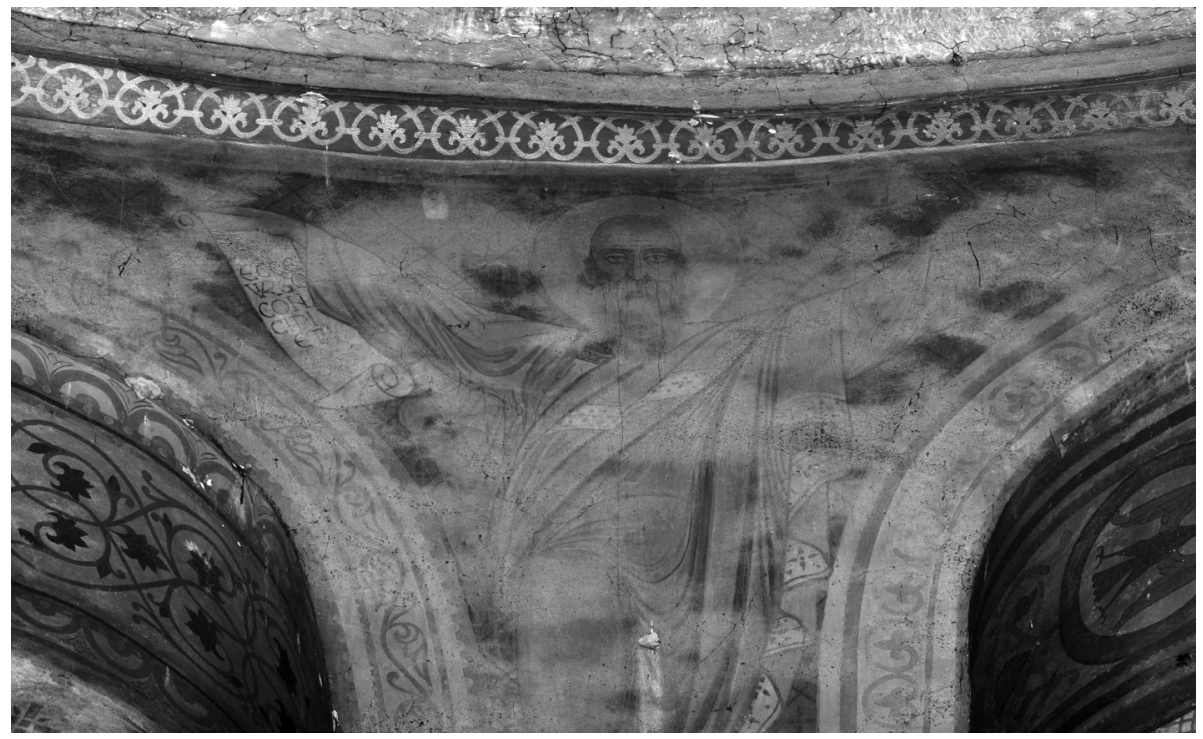

Fig. 5. - Le prophète Isaïe

(cl. CIFM-CESCM/J.-P. Brouard)

Sur le phylactère qu'il tient en sa main droite on lit :

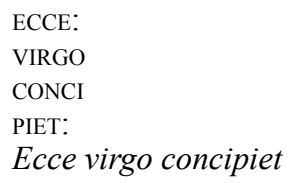

Voici qu'une vierge concevra [un fils qu'elle appellera Emmanuel]

35. Sermon 47, PL, XXXVIII, c. 299-301.

36. Commentaria in Ezechielem, PL, XXV, c. 331.

37. Raban Maur, Commentariorum in Ezechielem libri viginti, PL, CX, c. 845. 
«Le Seigneur donne un signe au roi Achaz : Écoutez, maison de David, (Isaïe 7:13)... Le Seigneur va vous donner un signe: Voici qu'une vierge concevra» (7:14). Cette annonce de la conception virginale et de la naissance de Jésus a été largement commentée par les exégètes, ainsi Jérôme, Rémi d'Auxerre, Rupert de Deutz ${ }^{38}$, et particulièrement Hugues de Saint-Victor qui lui consacre un traité ${ }^{39}$. C'est le verset que prononce Isaïe dans le Sermon contre les Juifs de Quodvultdeus, repris dans le Mystère d'Adam. Il est cité par Matthieu 1:23, lorsqu'il parle de la conception virginale de Marie. Il fait partie de la liturgie de l'Annonciation et de l'Avent ${ }^{40}$. L'annonce prophétique est citée à de nombreuses reprises dans les inscriptions qui accompagnent des œuvres médiévales, déjà vers 800 sur un ivoire de la Bodleian Library à Oxford, mais surtout au XII ${ }^{\mathrm{e}}$ siècle, Moissac, Vienne, Lyon (cathédrale, Saint-Martin-d'Ainay) en France, cathédrales de Crémone, Plaisance, Salerne, Venise, comme à Castell'Arquato ou Tivoli en Italie, Orense en Espagne, Berlin, Hanovre en Allemagne, Millstatt en Autriche, basilique de la Nativité à Bethléem, et encore au XIII ${ }^{e}$ siècle à Moulins, Goslar, Hildesheim, Worms ${ }^{41}$.

Aux écoinçons du côté sud sont représentés, de gauche à droite, Daniel et Jérémie.

Le nom de Daniel (fig. 6) est écrit de part et d'autre de sa tête:

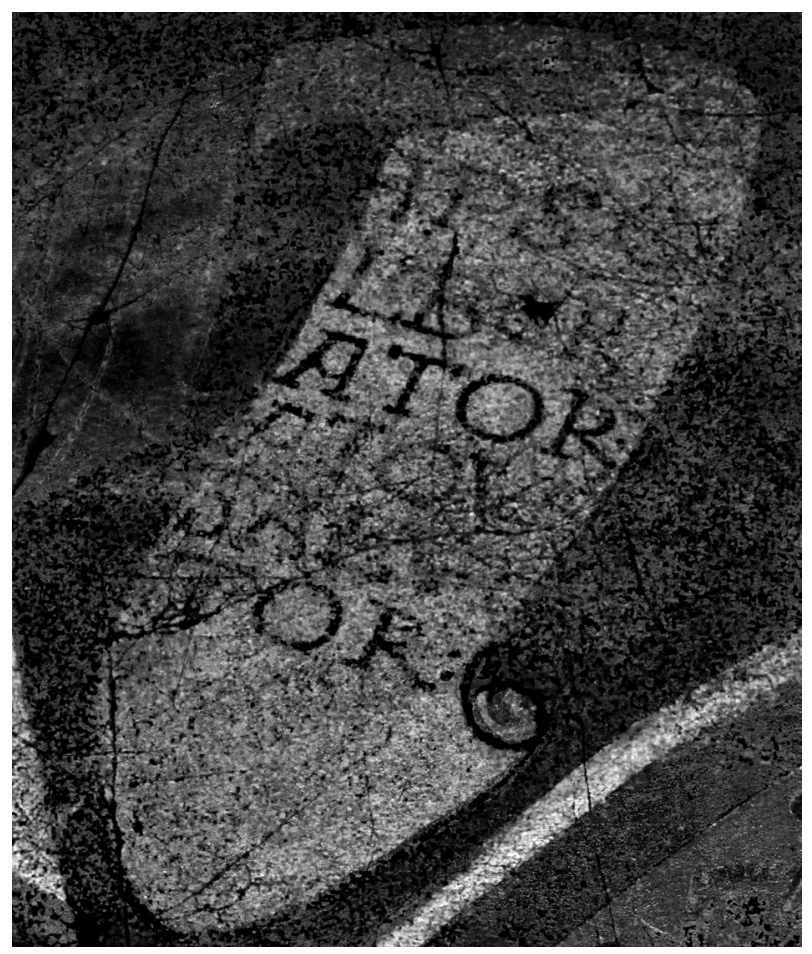

Fig. 6. - Le prophète Daniel

(cl. CIFM-CESCM/J.-P. Brouard)

38. JÉrôme, $P L, \mathrm{XXV}$, c. 107-109; RÉmi D’AuXerre (œuvre éditée sous le nom d'HAYMOn), Ibid. CXVI, c. 761-762; RuPERT, Ibid., CLXVII , c. 1306.

39. Ibid., CXCVI, c. 601-666, De Emmanuuele libri duo.

40. Corpus antiphonalium officii, R.-J. HeSBERT et R. PrÉvost (éd.), vol. III, Rome, Herder (Rerum ecclesiasticarum documenta. Series major. Fontes, IX et X), 1968, p. 191, n²257, et vol. IV, 1970, p. 159, nº 6620.

41. Poitiers, CESCM, fichier que j'ai établi pour les citations bibliques dans les inscriptions. On pourra se reporter également à la thèse de Françoise GAY, Les prophètes (épigraphie), thèse de $3^{\mathrm{e}}$ cycle sous la direction de R. FAVREAU, Poitiers, 1980, et à son article, «Les prophètes du XI ${ }^{\mathrm{e}}$ au XIII ${ }^{e}$ siècle, épigraphie», paru dans les CCM, XXX, 1987, p. 357-367. 
DAN IEL

Daniel

Sur le phylactère qu'il tient en sa main gauche on lit:

IPSE
LIBER
ATOR:
ATQVE
SALVA
TOR:
Ipse liberator atque salvator
Il est celui qui délivre et qui sauve

Le roi Darius avait signé un acte interdisant d'adresser une prière à quiconque, dieu ou homme, autre que lui, sous peine d'être jeté dans la fosse aux lions. Mais Daniel continua à prier Dieu trois fois par jour. Darius le fait jeter dans la fosse aux lions, en lui disant: «Ton Dieu que tu as servi avec persévérance, c'est lui qui te sauvera». Daniel sort indemne de la fosse. Le roi Darius écrit à tous les peuples : "Que les gens tremblent et frémissent devant le Dieu de Daniel. Il est le Dieu vivant il perdure à jamais, son royaume ne sera pas détruit et son empire n'aura pas de fin. Il sauve et délivre (verset 27 pour la Vulgate, 28 pour la Bible de Jérusalem), opère signes et merveilles aux cieux et sur la terre; il a sauvé Daniel du pouvoir des lions » (Daniel, 6) ${ }^{42}$.

Saint Jérôme rappelle que Daniel avait interprété le songe de Nabuchodonosor, qui avait alors reconnu «le roi du ciel, dont toutes les œuvres sont vérité» (4:31-34), et il met en rapport l'épisode de Daniel jeté dans la fosse aux lions par Darius : Daniel est épargné et le roi Darius reconnaît que le Dieu de Daniel est le Dieu vivant, libérateur et sauveur. «Ces signes, ajoute-t-il, ont eu lieu par les serviteurs de Dieu auprès des nations barbares, afin que soient prêchés le culte et la religion du Dieu unique $»^{43}$. Le rapprochement fait par Jérôme de Nabuchodonosor et de Darius est intéresssant, car le Sermon contre les Juifs de Quodvultdeus a retenu Nabuchodonosor comme un témoin païen du Dieu d'Israël, que l'on retrouve dans la frise de NotreDame-la-Grande avant les prophètes. Daniel dans la fosse aux lions a été représenté sur un chapiteau de la façade de l'église Saint-Porchaire de Poitiers.

Au dernier écoinçon, avant la sibylle tiburtine, on a le dernier des quatre grands prophètes, Jérémie (fig. 7), avec son nom inscrit de part et d'autre de sa tête:

HIERE MIAS

Hieremias

Jérémie

Sur le phylactère qu'il tient en sa main gauche on peut lire:

GERMI
NARE:
FACIA
M:DAVI
D:GERM
EN:IVS
TITIAE
Germinare faciam David germen justitice
Je ferai germer pour David un germe de justice

42. Il a fallu que le photographe Jean-Pierre Brouard travaille minutieusement la photographie pour faire ressortir le texte qui était totalement illisible.

43. JÉrôME, Commentaria in Danielem, PL, XXV, c. 527. 


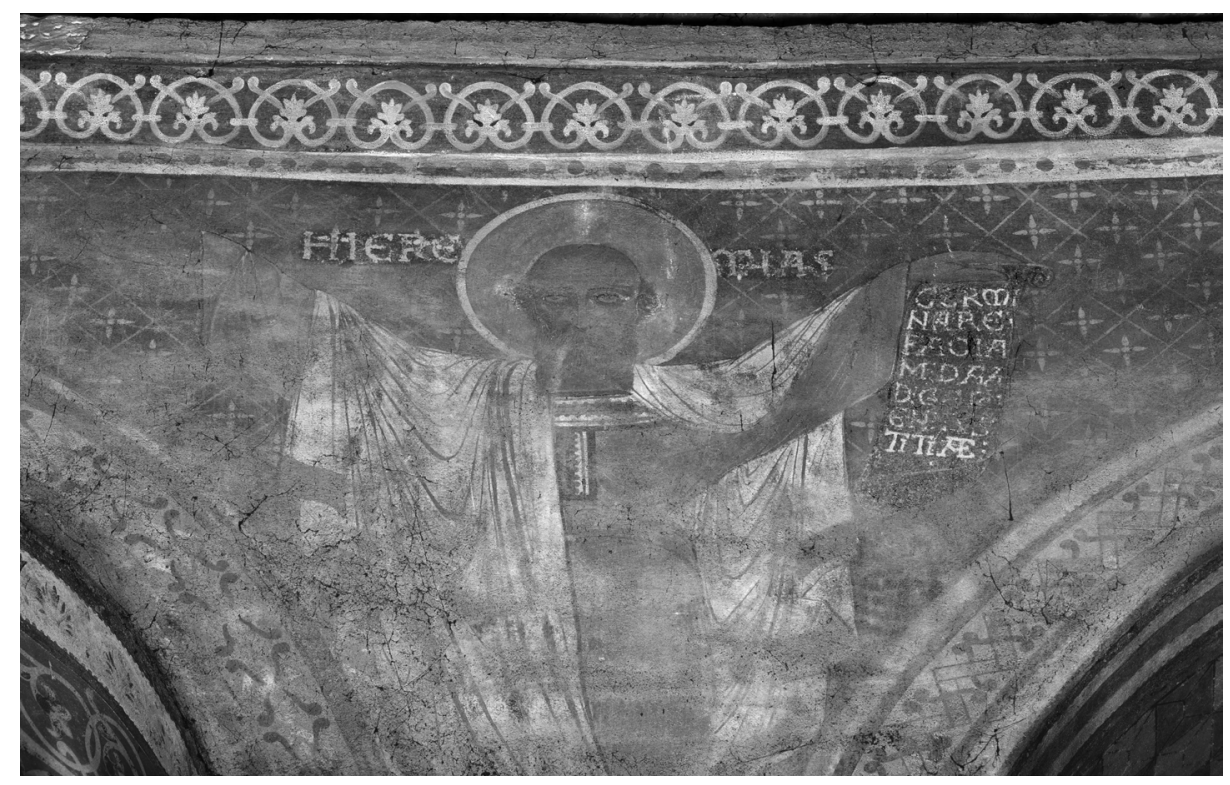

Fig. 7. - Le prophète Jérémie

(cl. CIFM-CESCM/J.-P. Brouard)

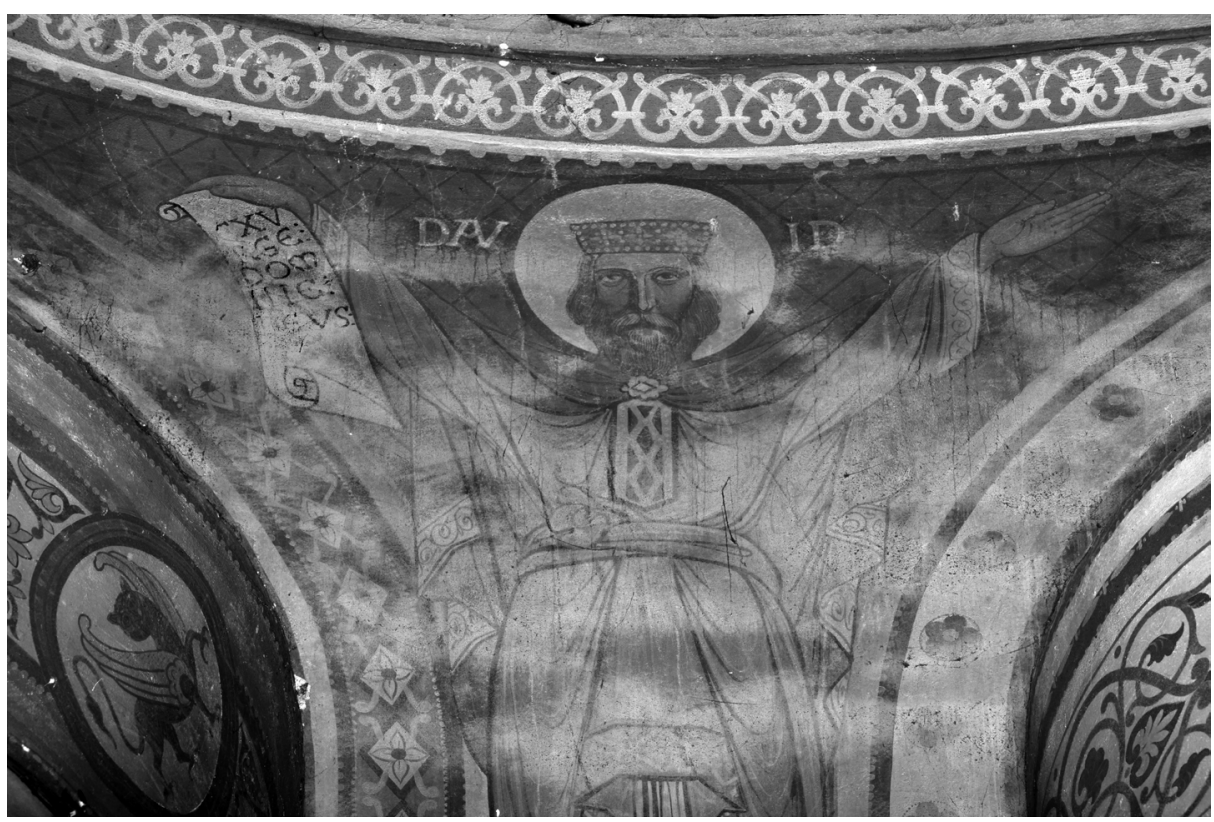

Fig. 8. - Le roi David

(cl. CIFM-CESCM/J.-P. Brouard) 
En mettant côte à côte Daniel (chapitre 6) et Jérémie (33:15), l'auteur du programme a peut-être pensé à Samuel 17:37, où David dit: «Le Seigneur m'a arraché de la griffe du lion». Plus sûrement est évoqué ici le début de l'Évangile selon saint Matthieu: «Généalogie de Jésus Christ, fils de David, fils d'Abraham» (1:1). Dans son commentaire sur Matthieu, Hilaire de Poitiers a longuement commenté ce début de l'Évangile: en recensant la lignée paternelle qui partait de Juda, Matthieu fait ressortir la succession royale, et montre Jésus éternellement roi; Marie était également de la tribu de Juda ${ }^{44}$. Jésus est dit «fils de David» neuf fois chez Matthieu, trois fois chez Marc, quatre fois chez Luc, Jean 7:42 dit le Christ de la souche de David (ex semine David), comme l'écrira Paul à deux reprises (Romains 1:3;2 Timothée 2:8).

Cette citation de Jérémie 33:15 est évoquée dans la liturgie de l'Annonciation et de l'Avent : Ecce dies venient, ait Dominus, et suscitabo David germen justum, et regnabit rex et sapiens erit, et faciet judicium et justiciam in terra ${ }^{45}$. Raban Maur écrit: «Le Seigneur fait germer pour David un germe de justice, c'est-à-dire le Christ, qui descendra de sa lignée, qui fera jugement et justice sur la terre ${ }^{46}$.

On notera aussi que la citation d'Isaïe 7:14 sur la conception virginale de l'Emmanuel était adressée à la «maison de David».

Les deux plus célèbres rois de Juda, David et Salomon, sont représentés dans les écoinçons de l'axe du rond-point, David (fig. 8) à droite, c'est-à-dire à côté de Jérémie qui annonçait Jésus comme de la lignée de David. Son nom est de chaque côté de sa tête:

DAV ID

Sur le phylactère qu'il tient en sa main droite on lit :

EXV

RGE:

DOM

INE:

DEVS

Exurge, Domine Deus

Dresse-toi, Seigneur Dieu

David est considéré comme l'auteur des psaumes. On trouve à quatre reprises dans les psaumes la supplication Exurge Domine $(7: 7 ; 10: 12 ; 16: 13 ; 43: 26$ ), mais seulement dans le psaume 10:12 (Bible de Jérusalem 9-10:12) la forme Exsurge Domine Deus, "Dresse-toi, Seigneur, Dieu lève ta main», que l'on trouve employée dans la liturgie ${ }^{47}$. Tous les grands auteurs chrétiens, à commencer par Hilaire de Poitiers, ont commenté les psaumes. Le passage choisi sur le phylactère ne semble pas avoir une intention particulière. David annonce le Seigneur Dieu, qui est de sa lignée, comme le rappelle Jérémie sur l'écoinçon voisin.

David est aussi un des témoins avancés dans le Sermon contre les Juifs qu'à cette époque on pense être d'Augustin, David sanctus, testis fidelis, «saint David, fidèle témoin».

Teste David cum sibylla dira l'hymne Dies irce, attribuée à Thomas de Celano mais connue dès la fin du $\mathrm{XII}^{\mathrm{e}}$ siècle.

Sur l'écoinçon à gauche de l'axe du chœur est figuré Salomon (fig. 9), avec son nom inscrit de chaque côté de sa tête:

44. Hilaire de Poitiers, Sur Matthieu, J. Doignon (éd. et trad.), Paris, Le Cerf, 1978 (Sources chrétiennes, 254), p. 91-99.

45. Corpus antiphonalium... (op. cit. n. 40), III, p. 185, n² 2506, et IV, p. 150, n 6583.

46. Raban Maur, Expositio super Jeremiam, PL, CXI, c. 1065.

47. Corpus antiphonalium... (op. cit. n. 40), IV, p. 178, n 6706. 


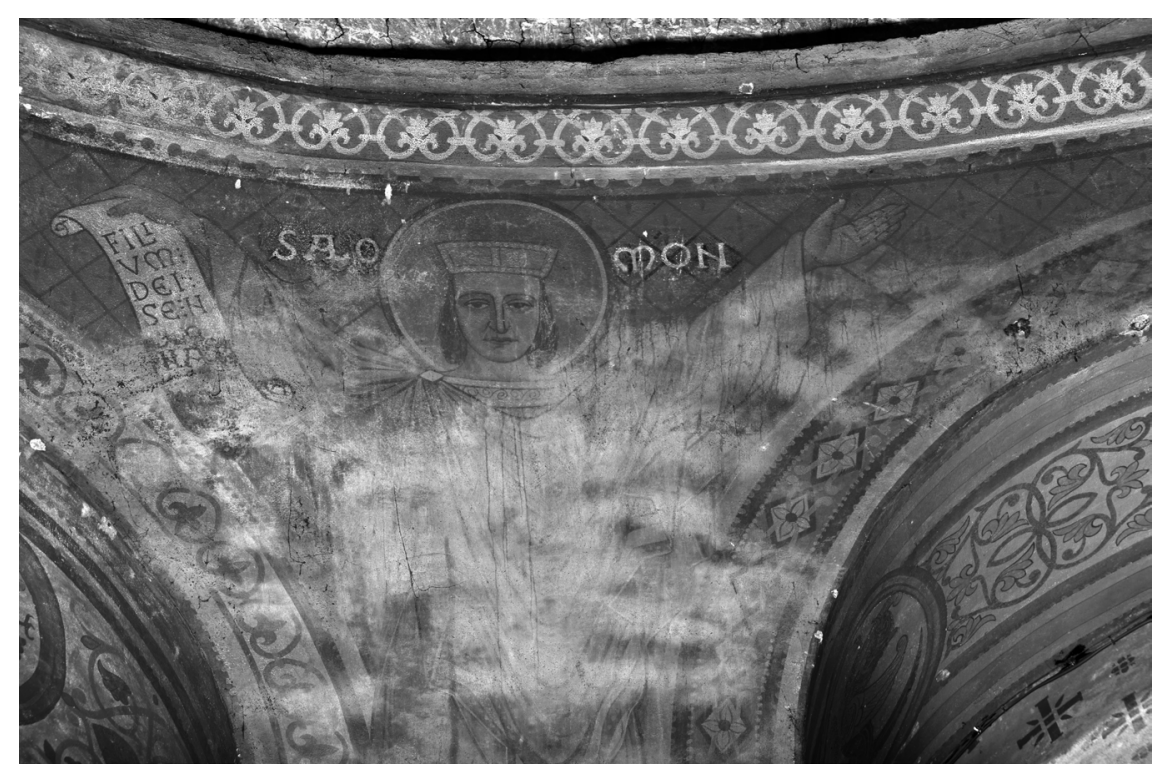

Fig. 9. - Le roi Salomon

(cl. CIFM-CESCM/J.-P. Brouard)

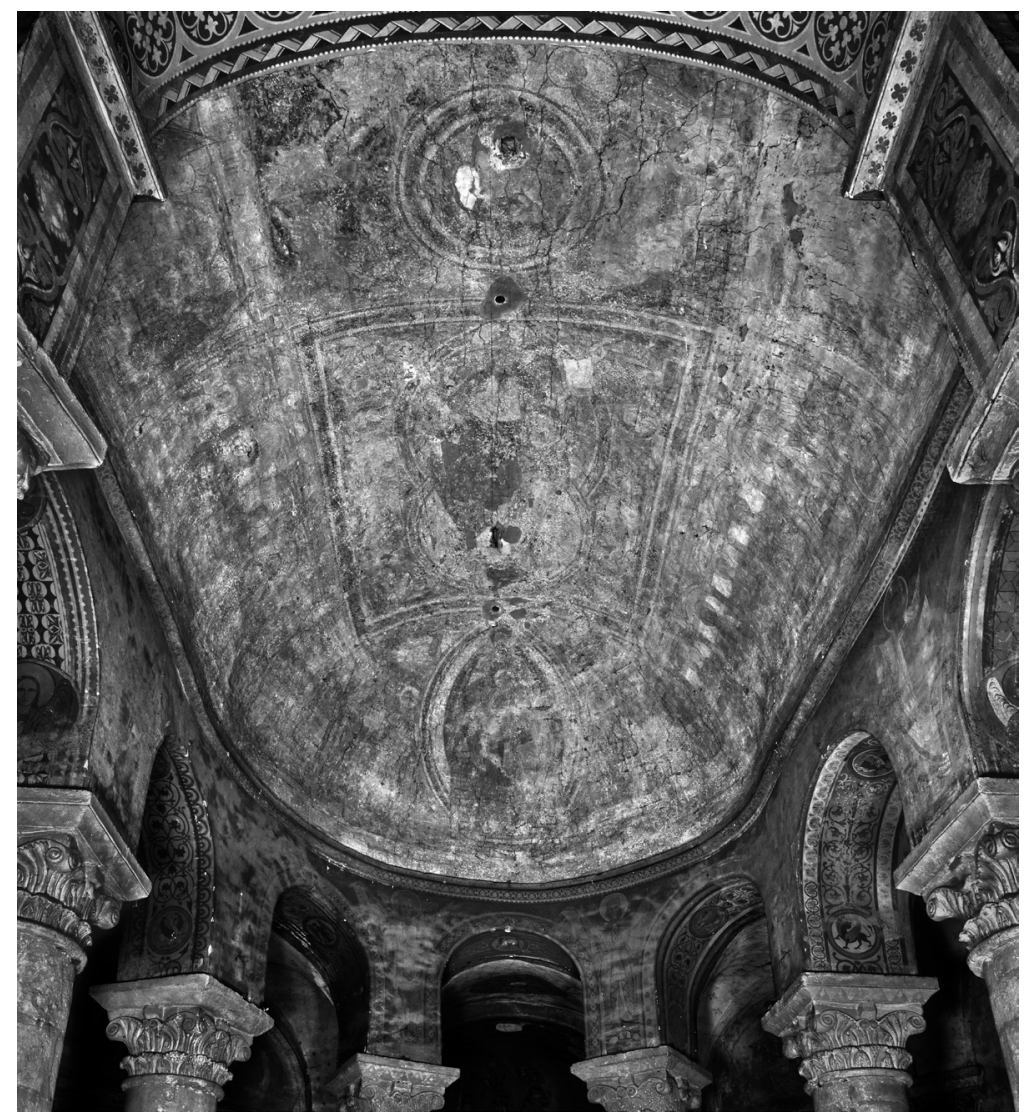

Fig. 10. - La voûte et le rond-point

(cl. CIFM-CESCM/J.-P. Brouard) 


\begin{abstract}
SALO MON
Sur le phylactère qu'il tient en sa main droite on lit :

FILI

VM:

DEI:

SE:N

$\mathrm{OM}$

INAT:

Filium Dei se nominat
\end{abstract}

«Il se nomme lui-même Fils de Dieu»

C'est une citation du livre de la Sagesse 2:13. L'auteur de ce livre est pensé être Salomon, qui est clairement désigné au chapitre 9, versets 7-8 et 12 ; le livre s'appelle en grec «Sagesse de Salomon».

Si Jésus est dit par le Père son Fils bien aimé, au Baptême et à la Transfiguration, s'il est dit Fils de Dieu à de nombreuses reprises dans les évangiles, ce n'est qu'au moment de la Passion qu'on trouve l'expression Filius Dei sum, «Je suis le Fils de Dieu» (Jean 10:36). On trouve la formule même de la Sagesse dans la liturgie du dimanche des Rameaux, au troisième nocturne, et au cours de la semaine sainte: Dixerunt impii apud se, non recte cogitantes : circumveniamus justum...; promittit se scientiam Dei habere, Filium Dei se nominat, et gloriatur Patrem se habere Deum ${ }^{48}$.

Le Sermon contre les Juifs ne mentionne pas Salomon, mais le Mystère d'Adam le met en scène aussitôt après David ${ }^{49}$.

Le programme des peintures murales du rond-point apparaît très élaboré. Comme la frise de la façade il est directement inspiré par le Sermon contre les Juifs de Quodvultdeus à l'époque attribué à Augustin, le grand maître à penser du Moyen Âge, et par le Mystère d'Adam, le drame liturgique qui a d'abord été représenté dans le chœur au moment de Noël, avant d'émigrer sur les parvis. Le Sermon met en scène les sibylles, Isaïe, Jérémie, Daniel, David, Nabuchodonosor, pour convaincre les Juifs «qui nient le Fils de Dieu». Le Mystère d'Adam ignore les sibylles, ajoute Salomon. Les peintures murales du rond-point mettent en scène deux sibylles, les quatre grands prophètes, en comprenant Ezéchiel pour équilibrer le programme, les rois David et Salomon. Si Nabuchodonosor n'est pas nommé, ce sont les paroles mêmes d'un autre roi païen Darius qui sont inscrites sur le phylactère de Daniel, le prophète qui avait aussi rapporté la reconnaissance par Nabuchodonosor du Dieu d'Israël.

Les peintures du rond-point annoncent, par les prophétesses païennes, les grands prophètes, les rois David et Salomon, Jésus Fils de Marie et Fils de Dieu glorifié dans les peintures du cul-de-four et de la voûte de l'abside (fig. 10). La grande figure du Christ en gloire de la voûte encadrée par deux mandorles, est évidemment celle du Christ en sa divinité. Au rond-point, la sibylle Érythréenne dit précisément que «Jésus Christ» est «le Fils de Dieu Sauveur». Salomon rapporte: «Il se nomme lui-même Fils de Dieu». Ézéchiel rappelle qu'il est le juste juge. Le roi Darius, par le livre de Daniel, le dit le Dieu vivant, «qui sauve et qui libère». Le Fils de Dieu a voulu prendre la nature humaine, le «Verbe s'est fait chair et il a habité parmi nous». Il est né d'une femme, et au cul-de-four il est représenté, dans une gloire en amande, sur les genoux de sa Mère, Marie qui est «plus grande» que toutes les autres femmes, Sancta Marie Major, Notre-Dame la Grande. Au rond-point la Sibylle Tiburtine annonce la naissance du Christ à Bethléem, Isaïe prophétise la conception virginale d'Emmanuel, «Dieu avec nous », Jérémie indique que le Messie sera de la lignée de David, et David est représenté en confirmation de la prophétie. Il s'agit bien ici d'un programme très élaboré, en annonce du programme de la voûte de l'abside. Jésus ressuscité reprend les pèlerins en route pour Emmaüs: «Esprits sans intelligence, lents à croire tout ce qu'ont annoncé les prophètes... Et commençant par Moïse et parcourant tous les prophètes il leur interpréta dans toutes les Écritures ce qui le concernait» (Luc 24:25-27). Depuis la Résurrection, il faut lire l'Ancien Testament comme l'annonce du Nouveau Testament.

48. Corpus antiphonalium... (op. cit. n. 40), III, p. 155, n 2263, et IV, p. 118, n 6464 .

49. Le Mystère d'Adam... (éd. cit. n. 31), p. 84-85. 
Le programme iconographique et épigraphique du rond-point n'est pas seulement en relation avec les peintures murales de la voûte de l'abside. Il sera repris dans la frise de la façade, avec les mêmes sources, mais avec les textes mêmes du Sermon contre les Juifs, pour illustrer l'annonce de l'Incarnation dès l'Ancien Testament, et représenter à la suite la réalisation de ces annonces vétéro-testamentaires, Annonciation, Visitation, Nativité, pour rendre gloire à Marie, devenue, par son humble soumission à l'annonce de Gabriel, la mère de Dieu, Sancta Maria Major, Notre-Dame la Grande.

Il faudra désormais prendre en compte le programme iconographique et épigraphique très élaboré des peintures murales du rond-point dans toute étude sur Notre-Dame-la-Grande.

Robert FAVREAU

Université de Poitiers/CESCM 
